\title{
Live imaging of stem cell and progeny behaviour in physiological hair-follicle regeneration
}

\author{
Panteleimon Rompolas ${ }^{1}$, Elizabeth R. Deschene ${ }^{1 *}$, Giovanni Zito ${ }^{1 *}$, David G. Gonzalez ${ }^{2}$, Ichiko Saotome ${ }^{1}$, Ann M. Haberman ${ }^{2}$ \\ \& Valentina Greco ${ }^{1}$
}

Tissue development and regeneration depend on cell-cell interactions and signals that target stem cells and their immediate progeny ${ }^{1}$. However, the cellular behaviours that lead to a properly regenerated tissue are not well understood. Using a new, noninvasive, intravital two-photon imaging approach we study physiological hair-follicle regeneration over time in live mice. By these means we have monitored the behaviour of epithelial stem cells and their progeny ${ }^{2-4}$ during physiological hair regeneration and addressed how the mesenchyme $e^{5}$ influences their behaviour. Consistent with earlier studies ${ }^{6}$, stem cells are quiescent during the initial stages of hair regeneration, whereas the progeny are more actively dividing. Moreover, stem cell progeny divisions are spatially organized within follicles. In addition to cell divisions, coordinated cell movements of the progeny allow the rapid expansion of the hair follicle. Finally, we show the requirement of the mesenchyme for hair regeneration through targeted cell ablation and long-term tracking of live hair follicles. Thus, we have established an in vivo approach that has led to the direct observation of cellular mechanisms of growth regulation within the hair follicle and that has enabled us to precisely investigate functional requirements of hair-follicle components during the process of physiological regeneration.

Although stem cells and their immediate progeny are critical for tissue regeneration, we still lack knowledge concerning the discrete sequential steps that lead to proper tissue regeneration. Available methods to address these questions during regeneration are largely static and provide only snapshots of this highly dynamic process. An alternative approach is to continuously visualize stem cells and their progeny throughout physiological regeneration. Recent technological advances have enabled stem cell imaging in mammalian tissues in vivo, such as haematopoietic stem cells in the bone marrow ${ }^{7,8}$. However, these studies are inherently invasive and therefore it is not possible to monitor the same stem cells for long periods (days to weeks) using these methods.

To study a physiological tissue regeneration process, we established a method to image entire skin hair follicles over time in live mice. The skin is a suitable tissue for live imaging owing to its accessibility ${ }^{9,10}$. In addition, hair follicles continuously regenerate through alternating rest (telogen) and growth (anagen) phases (Supplementary Fig. 1), enabling us to study stem cell behaviours during physiological regeneration. Furthermore, hair follicles are anatomically well defined and the location of different cell types is well characterized ${ }^{2,6,11,12}$. During the quiescent phase, two populations coexist in the lower portion of the hair follicle; the stem cells, which are localized in a specialized region called the bulge, and their immediate progeny within the hair germ (Fig. 1a, b). The stem cell progeny is in contact with a mesenchymal group of cells—called dermal papilla (Fig. 1a) — that is able to induce hair regeneration (Supplementary Fig. 1) ${ }^{5}$. Our recent work has shown that signals released by the mesenchyme activate the progeny at the beginning of the hair regeneration cycle $^{6}$. Thus, hair regeneration initially relies mainly on the expansion of the progeny and not of the stem cells. Subsequently, stem cells in the bulge will slowly contribute to hair regeneration over time ${ }^{6,12-14}$.

To visualize directly the hair-follicle stem cells and progeny, we used a transgenic mouse line that marks all of the epithelial nuclei in the skin by expressing a fusion protein of histone $\mathrm{H} 2 \mathrm{~B}$ with green fluorescent protein (GFP) driven by the keratin 14 promoter (K14H2BGFP) ${ }^{3,6}$. This transgenic mouse provides a strong nuclear signal that can resolve individual cells. Using a two-photon laser scanning microscope we acquired serial optical sections from the skin of anaesthetized 3-week-old mice (Supplementary Fig. 2 and Supplementary movie 1), which enabled us to visualize the entire hair follicle and identify the location of the stem cells and their progeny based on their distinct morphological features (Fig. 1b and Supplementary Fig. 3).

During the early stages of regeneration, proliferation is first initiated in the stem cell progeny, which undergo a rapid downward expansion (Supplementary Fig. 1). We asked whether this asymmetrically organized growth of the hair follicle relies on spatial regulation of processes such as cell divisions. To test this idea, we performed time-resolved imaging of hair follicles at the beginning of the hair regeneration cycle (around postnatal day 22 (P22)) to visualize cell proliferation over several hours. By these means, we have recorded several mitotic events with clear spatial and temporal resolution (Fig. 1c and Supplementary movie 2). Systematic quantification of the location and axis of divisions from several recordings shows that most divisions occur in the lower part of the follicle at the interface between the progeny and the mesenchyme at the beginning of a new growth (Fig. 1d). At this stage, the axis of division is parallel to the long axis of hair follicle growth. A second, less pronounced, centre of growth was identified in the upper part of the progeny (Fig. 1d). Cell divisions were observed within the bulge even at lower frequencies, (Fig. 1d, e, and Supplementary movies 3 and 4). The axes of these bulge divisions varied, ranging from perpendicular to parallel with respect to the long axis of hair follicle growth (Fig. 1d, e). Hair growth in the mouse is a fairly synchronized process at 3 weeks of age. To test whether this synchronization is the result of temporal regulation of cell divisions within the hair follicle, we quantified the time when cell divisions occurred within a single hair follicle and in relation to neighbouring hair follicles (at approximately P22). In some of our recordings, clustering was observed between cell divisions within one follicle as well as between adjacent follicles. However, these behaviours were not consistent in every recording (Supplementary Fig. 4 and Supplementary movie 2).

During regeneration, the lower part of the hair follicle undergoes a major reorganization as the mesenchyme transitions from being extruded from the epithelium to being encompassed by it ${ }^{15}$ (Supplementary Fig. 1). This led us to propose that changes in tissue architecture may rely on other cellular processes in addition to cell divisions. Analysing several of our recordings we observed that some follicles rapidly stretch downwards during this growth phase (at approximately P23; Fig. 2a,

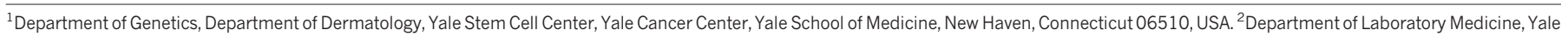
School of Medicine, New Haven, Connecticut 06510, USA.

*These authors contributed equally to this work. 

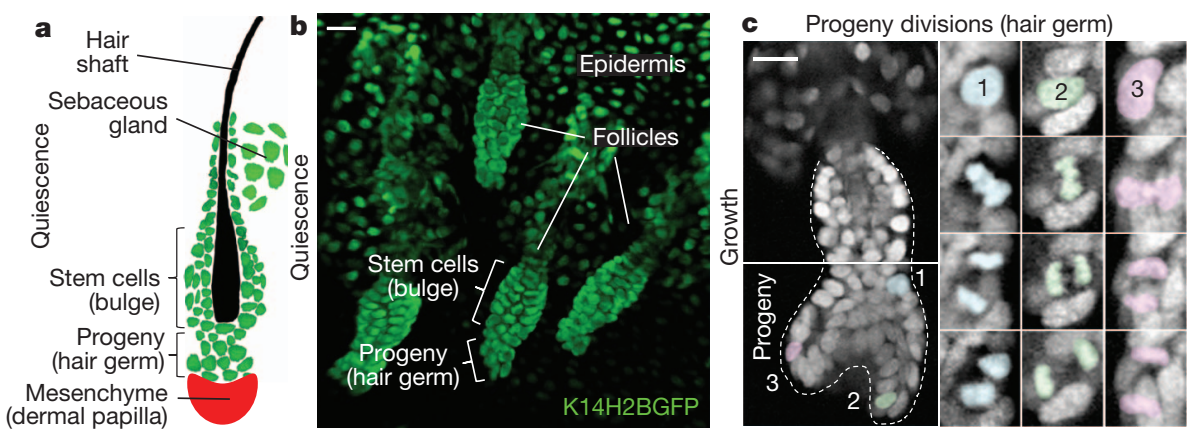

(dermal papilla)
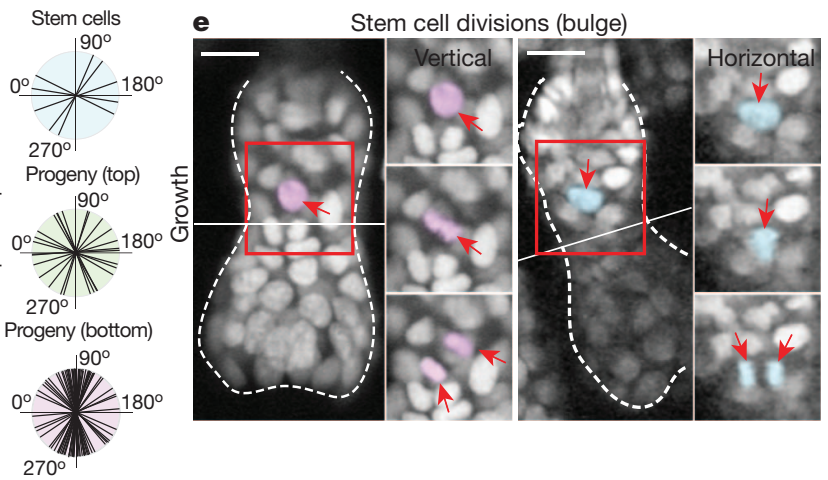

Figure $1 \mid$ Cell divisions are spatially regulated in the hair follicle at the beginning of a new growth. a, A hair follicle in quiescence. Distinct populations of cells that participate in hair regeneration, including stem cells, progeny and mesenchyme, reside in defined anatomical compartments of the hair follicle. b, A three-dimensional reconstruction of quiescent live hair follicles from serial optical sections acquired by two-photon laser scanning microscopy. Epithelial nuclei (green) are made visible using the $\mathrm{H} 2 \mathrm{~B}-\mathrm{GFP}$ fusion protein driven by the keratin 14 promoter (K14H2BGFP). c, An example of progeny cell division. A single optical section of a live hair follicle (left panel) and magnified views (right, insets) of three nuclei in the progeny compartment that undergo mitosis (right). d, The locations and axes of cell divisions were quantified from several hair follicles $(n=17)$ at early growth phase (anagen II). e, Two examples of vertical (left panel) and horizontal (right panel) stem cell divisions. A single optical slice of live hair follicles (left, insets) and magnified views of nuclei in the stem cell compartment (right, insets) undergoing mitosis. Red arrows, parental and daughter nuclei during mitosis. Time lapse between sequential frames is about $15 \mathrm{~min}(\mathbf{c})$ and $45 \mathrm{~min}(\mathbf{e})$. Scale bars, $20 \mu \mathrm{m}$.

Supplementary Fig. 5, and Supplementary movies 5 and 6). To test whether this tissue extension is the result of a morphological rearrangement of the tissue, we quantified the distance between adjacent nuclei within the stem cell or the progeny compartment. The distance between nuclei increased specifically within the progeny, which may account for the overall downwards extension of the hair follicle (Fig. 2a
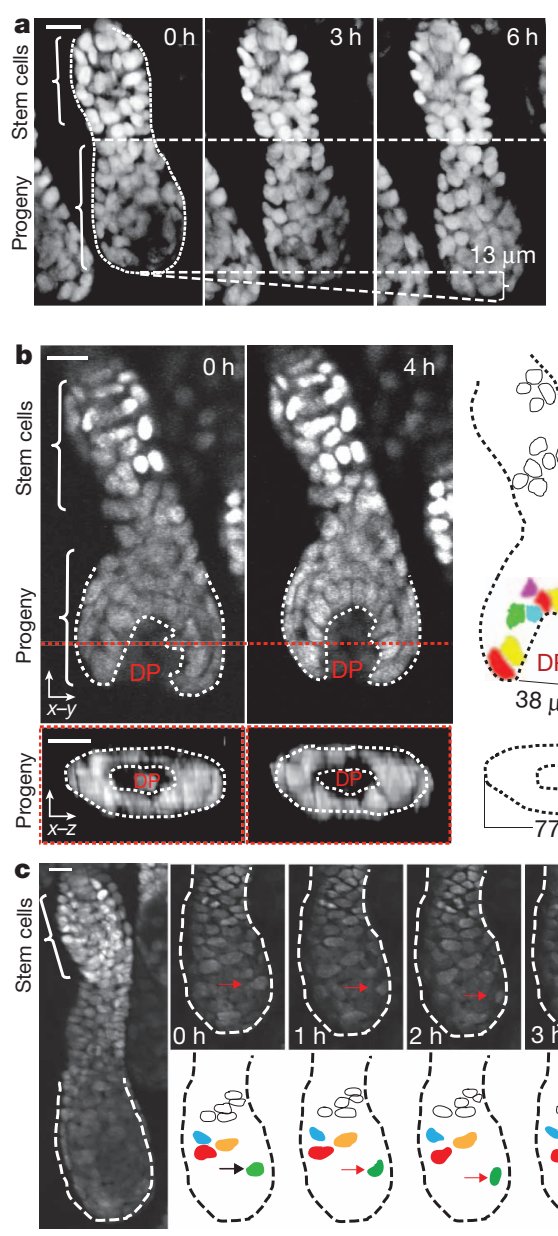
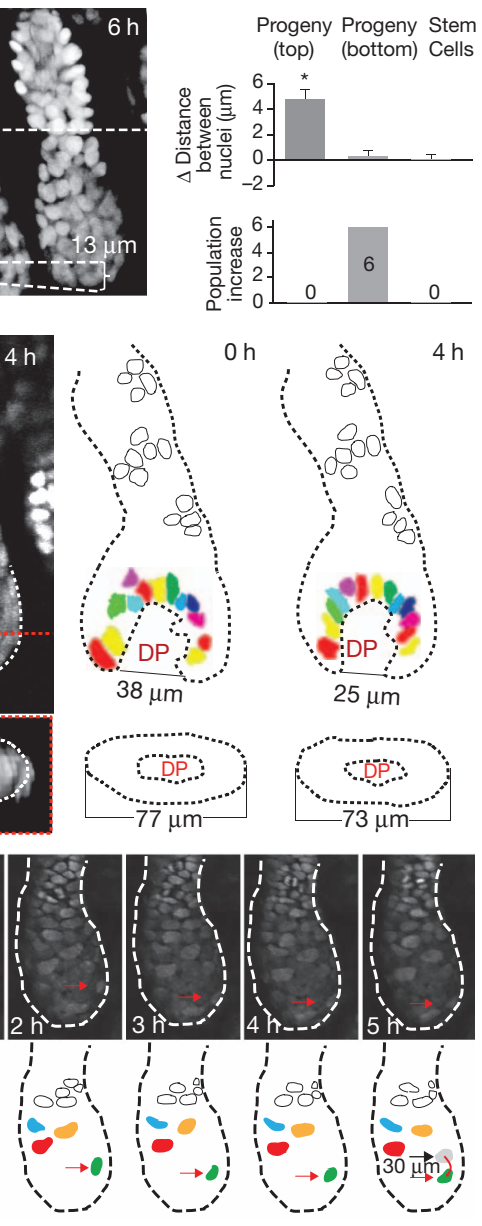

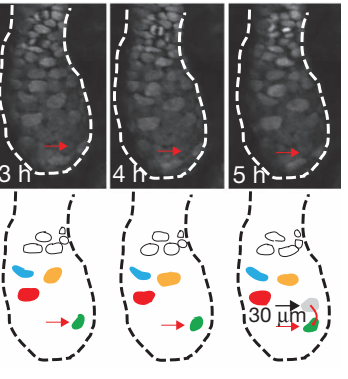

and Supplementary Fig. 5). This type of behaviour is reminiscent of morphogenetic movements observed during development in classical model organisms such as Drosophila or zebrafish (Danio) embryos ${ }^{16}$ and provides a previously unidentified mechanism of hair growth in addition to cell division.

To dissect further these novel dynamic processes, we analysed timeresolved optical sections at the coronal plane of the follicles where the epithelium interfaces the mesenchyme. During growth, in vivo recordings revealed a major reorganization of the epithelial stem cell progeny surrounding the mesenchymal dermal papilla. Over $4 \mathrm{~h}$ the nuclei transition from a disorganized pattern to a single row aligned around the mesenchyme (Fig. $2 \mathrm{~b}$ and Supplementary movie 7 ). In addition, the lower epithelial part of the follicle constricts as it encompasses the mesenchyme (Fig. 2b; 0 h versus $4 \mathrm{~h}$ ). This major epithelial nuclear reorganization occurs concurrently in adjacent follicles (Supplementary Fig. 6 and Supplementary movie 7). Furthermore, in more advanced growth stages, long-range migrations within the outer most layer (outer root sheath) of the lower hair follicle were observed (Fig. 2c and Supplementary movie 8). Recent data using lineage-tracing approaches have indicated that stem cells can migrate out of the bulge, either downwards towards the progeny or upwards towards the sebaceous gland (Fig. 1a) ${ }^{17,18}$. We did not observe downwards

Figure $2 \mid$ The stem cell progeny compartment undergoes morphological reorganization during growth. a, Downwards extension of follicles during growth. Optical sections at three consecutive time points ( $3 \mathrm{~h}$ apart) of a growing live hair follicle shows the progeny compartment extending downwards (left three panels). The increase in the distances between nuclei, and total cell numbers were quantified within the stem cells and the progeny compartments (approximately anagen II to IIIa) (right panel, data are expressed as mean \pm s.e.m., $n=13-20$; asterisk, $P<0.0001$; see also Supplementary Fig. 5). b, Reorganization of nuclei within the hair follicle. Two optical sections (left panels), corresponding traces and measurements (right panels) at the coronal and transverse planes ( $x y$ and $x z$ ) of the same follicle at time 0 and $4 \mathrm{~h}$, respectively (approximately anagen II to IIIa) (bottom panels). c, Downwards migration of nuclei in the hair follicle during growth. Single optical sections showing complete (left panel) and lower partial views (top panels) of a single hair follicle at successive time points, $1 \mathrm{~h}$ apart. Red arrows in the optical slices and corresponding traces (bottom panels) mark a nucleus that is moving downwards, covering a distance of $\sim 30 \mu \mathrm{m}$ within $5 \mathrm{~h}$ (approximately anagen IIIb). The position of the green nucleus at $0 \mathrm{~h}$ is shown in grey for comparison (bottom right panel). DP, dermal papilla. Scale bars, $20 \mu \mathrm{m}$. 
migrations of the stem cells to the progeny but captured a short upwards migration within the bulge stem cells (Supplementary Fig. 7 and Supplementary movie 9). Based on these data, we suggest that migratory events within the bulge may be temporally regulated or may take place at a much slower pace than we can resolve in the timeframe of our experiments (3-14 h). Taken together, our findings reveal new dynamic cellular processes adopted by the stem cells and their immediate progeny during physiological regeneration that would have been missed by conventional static analysis.

Epithelial-mesenchymal interactions are crucial for the development and regeneration of many organs such as limbs ${ }^{19}$. In the hair follicle, the mesenchymal dermal papilla is a key signalling centre, able to induce de novo hair-follicle formation after transplantation ${ }^{5}$. Moreover, previous work has identified mesenchymal signals, including signalling by FGF7 and FGF10, and BMP inhibitors, as regulators for the initiation of the hair regeneration $\mathrm{cycle}^{6,20}$. These and other data suggest that the mesenchyme is sufficient to induce hair regeneration. However, the requirement of the mesenchyme for initiation of hair regeneration has not been tested. To be able to selectively eliminate the mesenchyme, we set up a laser-induced cell-ablation approach to target fluorescently labelled dermal papilla cells using a Lef1RFP transgenic mouse (expressing red fluorescent protein under the control of a Lef1 promoter fragment) ${ }^{6,21}$ at the beginning of a new hair growth (at approximately P19; Fig. 3a and Supplementary Fig. 8). Analysis of the tissue immediately after laser ablation showed that the dermal papilla was disrupted, whereas adjacent tissues, such as the progeny or the overlying epidermis, remained undamaged (Supplementary Fig. 8). To assess the long-term effects of dermal papilla ablation on hair regeneration, we revisited the same hair follicles in separate imaging sessions, hours or even days apart (Fig. 3a). When the same hair follicles were observed at 4 and 7 days after ablation, follicles with ablated mesenchymal dermal papillae appeared quiescent (Fig. 3b). In contrast, surrounding follicles with intact mesenchyme grew at a normal pace (Fig. 3c, e). To determine whether the mesenchymal ablation altered the molecular and cellular composition of the hair follicle, skin samples containing mesenchymal ablated follicles were collected and analysed at 7 days post ablation. Histological and immunofluorescence data showed that the stem cells and their progeny maintain their molecular properties after dermal papilla ablation (Supplementary Fig. 9). Furthermore, the ablated follicles

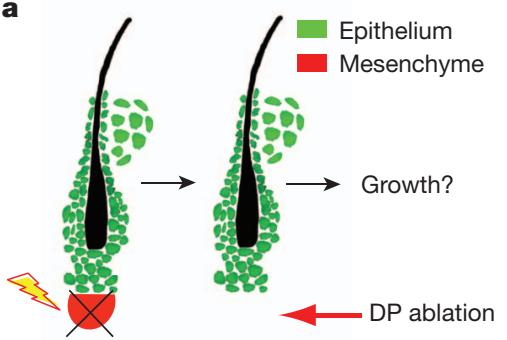

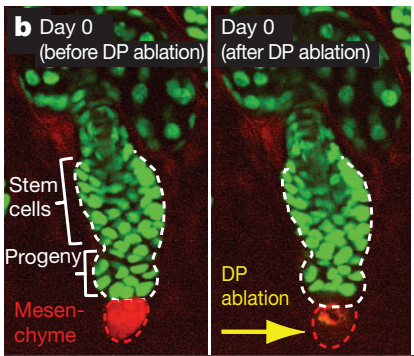

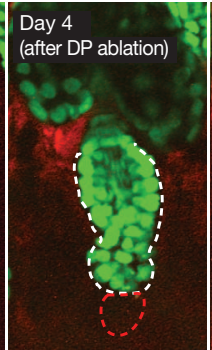

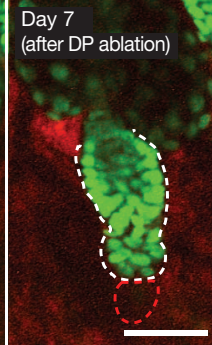
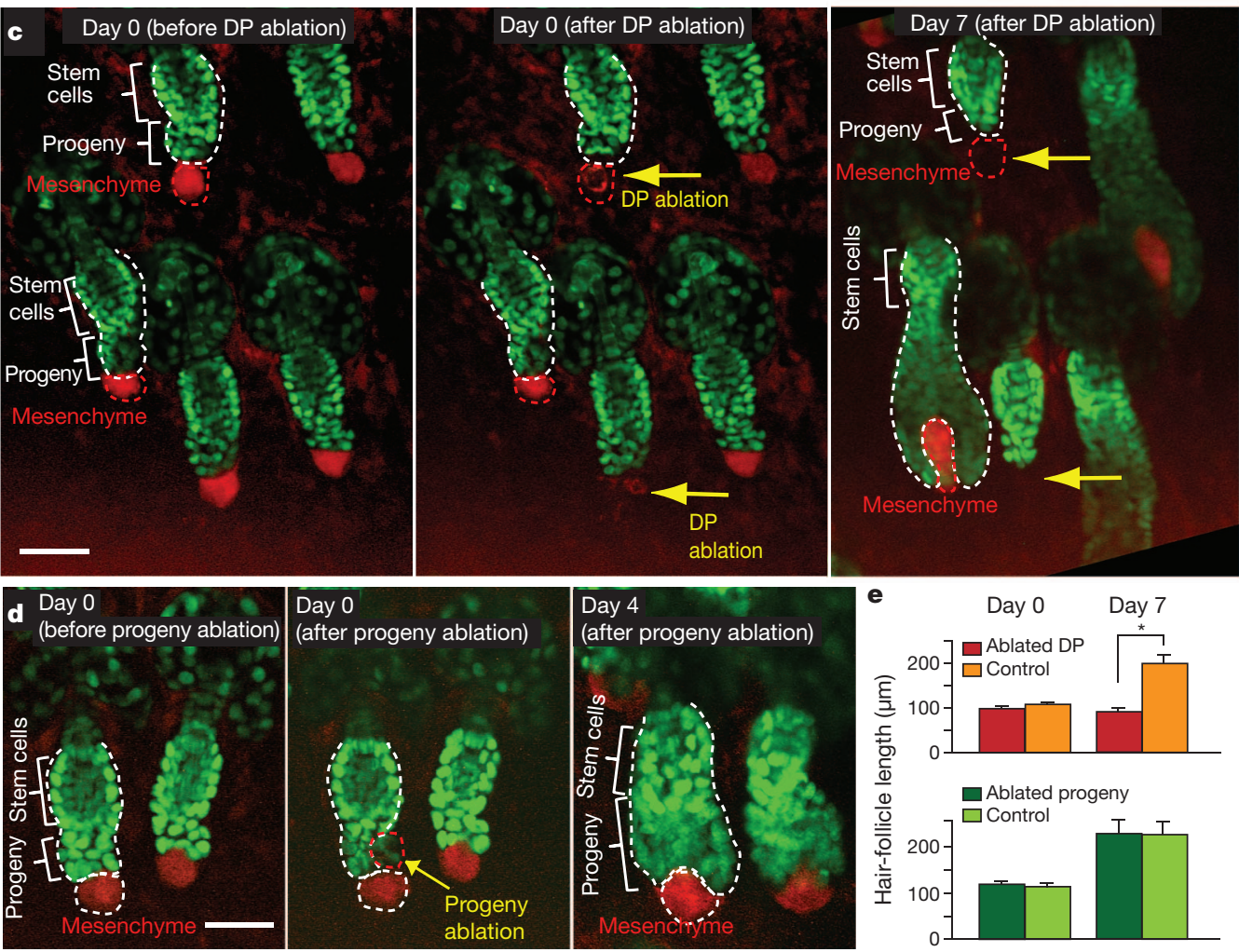

Figure 3 Ablation of the mesenchymal dermal papilla impairs initiation of hair regeneration. a, Set-up for the experiment using laser-induced cell ablation of dermal papilla cells to test the requirement of the mesenchyme for hair growth. b, High-magnification optical sections of four time points of live hair follicles whose dermal papilla was ablated (approximately telogen phase). c, Low-magnification optical sections of three time points show a group of follicles in which only a few follicles had the dermal papilla ablated (yellow arrows). d, Low-magnification optical sections of three time points show two follicles of which one had the progeny partially ablated (yellow arrow). e, Quantification of the growth of hair follicles (measured as the total length of the hair follicle) with ablated dermal papilla (top) or partially ablated progeny compartments (bottom) compared to intact control follicles. Data are expressed as mean \pm s.e.m. $(n=8-10$; asterisk, $P<0.0001)$. Scale bars, $50 \mu \mathrm{m}$. 


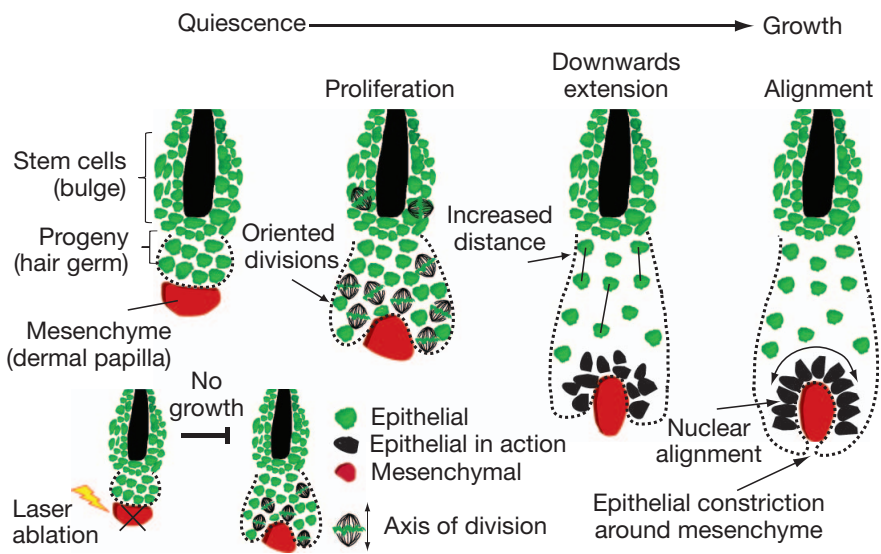

Figure $4 \mid$ The cellular mechanisms that participate in hair regeneration. During the initial stages of hair regeneration, the stem cell progeny is the first compartment that starts to proliferate. Cell divisions are also detected within the bulge, although the number of divisions is lower than in the stem cell progeny. Divisions in the progeny compartment are oriented along the axis of hair follicle growth, whereas orientations of divisions in the bulge are more random. The hair follicle undergoes a downwards extension in which the distance between nuclei increases within the progeny but not within the stem cell compartment. The epithelial nuclei surrounding the mesenchymal dermal papilla realign and constrict around the mensenchyme. Ablation of the mesenchyme results in impaired hair follicle growth.

retain the ability to host other tissue cell types such as melanocytes (Supplementary Fig. 9). To test whether failure to initiate hair growth was due to dermal papilla ablation rather than to collateral injury of the stem cell progeny, we targeted only cells within the progeny, leaving the dermal papilla intact. Hair follicles in which the progeny was injured recovered and grew at a similar rate to their surrounding uninjured follicles (Fig. 3d, e). These results indicate that the mesenchymal dermal papilla is required for hair growth initiation, and underscores the importance of epithelial-mesenchymal interactions for stem cell activation and regeneration.

In summary, we have imaged hair-follicle stem cells and their progeny in an uninjured living mammal, over a long period of time, with high spatial and temporal resolution. We have shown that hair regeneration involves a range of dynamic cellular behaviours and relies on the presence of the mesenchyme (Fig. 4). Given that components of the stem cell microenvironment are conserved across different tissues, our findings will probably be relevant to other tissues as well. More broadly, understanding how stem cell and progeny behaviour is regulated in physiological conditions may be necessary to advance our use of stem cells in regenerative medicine, and to uncover the cellular mechanisms that go awry in cancer and other diseases.

\section{METHODS SUMMARY}

K14H2BGFP, K14H2BGFP/Lef1RFP, K5tta and pTREH2BGFP transgenic mice were obtained from the Fuchs Laboratory and generated as previously described $^{3,21}$. All studies and procedures involving animal subjects were approved by the Institutional Animal Care and Use Committee of the Yale School of Medicine and were conducted in accordance with the approved animal handling protocol. Mice between 3 and 4 weeks of age were anaesthetized by intraperitoneal injection, and the skin in the ear and head was depilated. An adjustable mounting spatula was inserted though the ear canal, and the skin connecting the ear to the head was flattened and dry mounted with a coverslip. Mice were maintained on a heating stage and provided with vaporized anaesthetic through a nose cone for the course of the live imaging session.

All mice were imaged with a LaVision TriM Scope II (LaVision Biotec) twophoton laser scanning microscope using a $\times 20$ water immersion lens (N.A. 1.0; Olympus). Excitation (GFP, $940 \mathrm{~nm}$; RFP, $1040 \mathrm{~nm}$ ) and ablation $(900 \mathrm{~nm})$ were performed using a Chameleon Vision II (Coherent) laser. Three-dimensional stacks consisting of 50 optical sections in 2- $\mu \mathrm{m}$ steps along the $z$ axis, providing a depth of approximately $100 \mu \mathrm{m}$ below the surface of the skin, were acquired every $5 \mathrm{~min}$. Image processing, measurements, assembly and editing of time-lapse movies were performed using Image J (NIH Image) or Volocity (Perkin Elmer). An unpaired Student's $t$-test was applied in the two-group statistical analysis and $P$ values of less than 0.05 were considered significant. Histological and immunofluorescence analysis of frozen skin sections was performed following standard protocols as described previously ${ }^{6}$.

Full Methods and any associated references are available in the online version of the paper at www.nature.com/nature.

Received 12 December 2011; accepted 8 May 2012.

Published online 1 July 2012.

1. Morrison, S. J. \& Spradling, A. C. Stem cells and niches: mechanisms that promote stem cell maintenance throughout life. Cell 132, 598-611 (2008).

2. Cotsarelis, G., Sun, T.T. \& Lavker, R. M. Label-retaining cells reside in the bulge area of pilosebaceous unit: implications for follicular stem cells, hair cycle, and skin carcinogenesis. Cell 61, 1329-1337 (1990).

3. Tumbar, T. et al. Defining the epithelial stem cell niche in skin. Science $\mathbf{3 0 3}$, 359-363 (2004).

4. Oshima, H., Rochat, A., Kedzia, C., Kobayashi, K. \& Barrandon, Y. Morphogenesis and renewal of hair follicles from adult multipotent stem cells. Cell 104, 233-245 (2001).

5. Jahoda, C. A., Horne, K. A. \& Oliver, R. F. Induction of hair growth by implantation of cultured dermal papilla cells. Nature 311, 560-562 (1984).

6. Greco, V. et al. A two-step mechanism for stem cell activation during hair regeneration. Cell Stem Cell 4, 155-169 (2009).

7. Xie, Y. et al. Detection of functional haematopoietic stem cell niche using real-time imaging. Nature 457, 97-101 (2009).

8. Lo Celso, C. et al. Live-animal tracking of individual haematopoietic stem/ progenitor cells in their niche. Nature 457, 92-96 (2009).

9. Uchugonova, A., Hoffman, R. M., Weinigel, M. \& Koenig, K. Watching stem cells in the skin of living mice noninvasively. Cell Cycle 10, 2017-2020 (2011)

10. Ra, H. et al. In vivo imaging of human and mouse skin with a handheld dual-axis confocal fluorescence microscope. J. Invest. Dermatol. 131, 1061-1066 (2011).

11. Watt, F. M. \& Jensen, K. B. Epidermal stem cell diversity and quiescence. EMBO Mol. Med. 1, 260-267 (2009).

12. Fuchs, E. The tortoise and the hair: slow-cycling cells in the stem cell race. Cell 137, 811-819 (2009)

13. Greco, V. \& Guo, S. Compartmentalized organization: a common and required feature of stem cell niches? Development 137, 1586-1594 (2010).

14. Waghmare, S. K. et al. Quantitative proliferation dynamics and random chromosome segregation of hair follicle stem cells. EMBO J. 27, 1309-1320 (2008).

15. Müller-Röver, S. et al. A comprehensive guide for the accurate classification of murine hair follicles in distinct hair cycle stages. J. Invest. Dermatol. 117, 3-15 (2001).

16. Wallingford, J. B., Fraser, S. E. \& Harland, R. M. Convergent extension: the molecular control of polarized cell movement during embryonic development. Dev. Cell 2, 695-706 (2002).

17. Zhang, Y. V., Cheong, J., Ciapurin, N., McDermitt, D. J. \& Tumbar, T. Distinct selfrenewal and differentiation phases in the niche of infrequently dividing hair follicle stem cells. Cell Stem Cell 5, 267-278 (2009).

18. Petersson, M. et al. TCF/Lef1 activity controls establishment of diverse stem and progenitor cell compartments in mouse epidermis. EMBO J. 30, 3004-3018 (2011).

19. Rubin, L. \& Saunders, J. W. Jr. Ectodermal-mesodermal interactions in the growth of limb buds in the chick embryo: constancy and temporal limits of the ectodermal induction. Dev. Biol. 28, 94-112 (1972).

20. Plikus, M. V. et al. Cyclic dermal BMP signalling regulates stem cell activation during hair regeneration. Nature 451, 340-344 (2008)

21. Rendl, M., Lewis, L. \& Fuchs, E. Molecular dissection of mesenchymal-epithelial interactions in the hair follicle. PLOS Biol. 3, e331 (2005).

Supplementary Information is linked to the online version of the paper at www.nature.com/nature.

Acknowledgements We are grateful to A. Horwich, V. Reinke and A. Giraldez for critical discussion of the work. We thank S. King for feedback on the manuscript, and E. Fuchs for the K14H2BGFP, Lef1RFP and pTREH2BGFP mice and A. Glick for the K5tta mice. P.R. is supported by the James Hudson Brown - Alexander Brown Coxe postdoctoral fellowship. G.Z. is a New York Stem Cell Foundation-Druckenmiller Fellow. E.D. is supported by the Cell and Molecular Biology Training Grant of the National Institute of General Medical Sciences, US National Institutes of Health, Public Health Service. This work was supported in part by the American Skin Association, the American Cancer Society and the Yale Rheumatologic Disease Research Core Center grant NIH P30AR053495.

Author Contributions P.R. and V.G. designed experiments and wrote the manuscript, and P.R. performed the experiments and analysed the data. E.D. performed two-photon laser-scanning timelapses. G.Z. performed immunostainings. I.S. set up the mouse colonies and staining protocols. D.G. and A.H. assisted on initial intravital imaging set-up. D.G. performed data analysis.

Author Information Reprints and permissions information is available at www.nature.com/reprints. The authors declare no competing financial interests. Readers are welcome to comment on the online version of this article at www.nature.com/nature. Correspondence and requests for materials should be addressed to V.G. (valentina.greco@yale.edu). 


\section{METHODS}

Transgenic mice. K14H2BGFP, K14H2BGFP/Lef1RFP, K5tta and pTREH2BGFP transgenic mice were generated as previously described ${ }^{3,21}$. All studies and procedures involving animal subjects were approved by the Institutional Animal Care and Use Committee of the Yale School of Medicine and were conducted in accordance with the approved animal handling protocol.

In vivo imaging. Three-week-old mice were anaesthetized with intraperitoneal injection of ketamine and xylazine, and the skin around the head region was shaved using a mechanical trimmer and depilatory cream. The mouse was placed on a heated stage, and the head and the ear were supported by a custom-made stage. A glass coverslip was placed against the skin in the junction region between the head and the ear. Image stacks of the skin were acquired with a LaVision TriM Scope II (LaVision Biotec) microscope equipped with a Chameleon Vision II (Coherent) twophoton laser. A laser beam (at $940 \mathrm{~nm}$ for GFP and $1040 \mathrm{~nm}$ for RFP, respectively) was focused through a $\times 20$ water immersion lens (N.A. 1.0; Olympus) and scanned with a field of view of 0.25 to $0.5 \mathrm{~mm}^{2}$ at $600 \mathrm{~Hz}$. Serial optical sections were acquired in $2-3-\mu \mathrm{m}$ steps to image a total depth of $\sim 100 \mu \mathrm{m}$ of tissue in 5 -min intervals. Several phases covering the transition from quiescent to growth stages were analysed (telogen to anagen phases). Distinctive inherent landmarks in the skin were used to navigate back to the original field of view and visualize the same follicles in separate experiments. Anaesthesia was maintained throughout the course of the experiment with vaporized isofluorane delivered by a nose cone.

Three-dimensional two-photon laser ablation. Laser ablation was carried out with the same optics as for acquisition, essentially as described previously ${ }^{22}$. A 900-nm laser beam was used to scan a $10 \mu \mathrm{m}^{2}$ area and ablation was achieved using $25 \%$ laser power for $1 \mathrm{~s}$. Ablation parameters were adjusted according to the depth of the target $(30-80 \mu \mathrm{m})$.

Image analysis. Raw image stacks $(512 \times 512$ to $1024 \times 1024$ pixels in the $x-y$ plane and $2-3-\mu \mathrm{m}$ voxel depth; typically $30-50$ optical sections) were imported into Image (NIH Image) for further analysis. Optical planes from sequential time points were manually realigned to compensate for minor tissue drift during the time course of the acquisition. The contrast was adjusted accordingly and selected optical planes or $z$-projections of sequential optical sections were used to assemble time-lapse movies. Three-dimensional rendering and cell tracking was performed using the Volocity software package (Perkin Elmer).

Statistical analysis. Data are expressed as mean \pm s.e.m. An unpaired Student's $t$-test was used to analyse data sets with two groups. For all analyses, a $P$ value of less than 0.05 was accepted as indicating a significant difference. Statistical calculations were performed using the Prism software package (GraphPad).

Histology and immunolabelling. Sections frozen in Tissue-Tek OCT Compound (Sakura Finetek) were fixed with $4 \%$ formaldehyde for $10 \mathrm{~min}$ at room temperature $\left(22^{\circ} \mathrm{C}\right)$ and treated as described previously ${ }^{6}$. Antibodies were used at the following dilutions: Ncam (Chemicon, rat 1:100), CD34 (eBioscience, rat 1:50), Pcadherin (R\&D, goat 1:100) and Trp2 (Santa Cruz, goat 1:100).

22. Eiraku, M. et al. Self-organizing optic-cup morphogenesis in three-dimensional culture. Nature 472, 51-56 (2011). 